\title{
The mutational decay of male-male and hermaphrodite- hermaphrodite competitive fitness in the androdioecious nematode $C$. elegans
}

\author{
Shu-Dan Yeh ${ }^{1,2} \cdot$ Ayush Shekhar Saxena ${ }^{1} \cdot$ Timothy A. Crombie $^{1} \cdot$ Dorian Feistel $^{1,3} \cdot$ Lindsay M. Johnson $^{1} \cdot$ \\ Isabel Lam ${ }^{1} \cdot$ Jennifer Lam ${ }^{1} \cdot$ Sayran Saber $^{1}$ - Charles F. Baer ${ }^{1,4}$
}

Received: 28 April 2017 / Revised: 27 July 2017 / Accepted: 16 August 2017 / Published online: 14 November 2017

(C) The Genetics Society 2018

\begin{abstract}
Androdioecious Caenorhabditis have a high frequency of self-compatible hermaphrodites and a low frequency of males. The effects of mutations on male fitness are of interest for two reasons. First, when males are rare, selection on male-specific mutations is less efficient than in hermaphrodites. Second, males may present a larger mutational target than hermaphrodites because of the different ways in which fitness accrues in the two sexes. We report the first estimates of male-specific mutational effects in an androdioecious organism. The rate of male-specific inviable or sterile mutations is $\leqslant 5 \times 10^{-4} / \mathrm{generation}$, below the rate at which males would be lost solely due to those kinds of mutations. The rate of mutational decay of male competitive fitness is $\sim 0.17 \%$ /generation; that of hermaphrodite competitive fitness is $\sim 0.11 \%$ /generation. The point estimate of $\sim 1.5 \mathrm{X}$ faster rate of mutational decay of male fitness is nearly identical to the same ratio in Drosophila. Estimates of mutational variance $\left(\mathrm{V}_{\mathrm{M}}\right)$ for male mating success and competitive fitness are not significantly different from zero, whereas $\mathrm{V}_{\mathrm{M}}$ for hermaphrodite competitive fitness is similar to that of non-competitive fitness. Two independent estimates of the average selection coefficient against mutations affecting hermaphrodite competitive fitness agree to within two-fold, $0.33-0.5 \%$.
\end{abstract}

\section{Introduction}

Several species of nematodes in the genus Caenorhabditis, among them the well-known $C$. elegans, have evolved an androdioecious mating system in which self-fertilizing hermaphrodites are very common and males are very rare. In $C$. elegans, for example, the frequency of outcrossing (=male-female mating, because hermaphrodites cannot mate with each other) is thought to be on the order of

Electronic supplementary material The online version of this article (doi:10.1038/s41437-017-0003-8) contains supplementary material, which is available to authorized users.

Charles F. Baer

cbaer@ufl.edu

1 Department of Biology, University of Florida, Gainesville, FL, USA

2 Department of Life Sciences, National Central University, Taoyuan, Taiwan

3 Department of Biology, Emory University, Atlanta, GA, USA

4 University of Florida Genetics Institute, Gainesville, FL, USA
$1-0.1 \%$ (Andersen et al. 2012). Dioecy is ancestral in the genus and most species in the genus are dioecious, although androdioecy has evolved independently at least three times (Kiontke et al. 2011). Moreover, at least in C. elegans, androdioecy appears to have evolved fairly recently $\left(\sim 10^{6}\right.$ generations or less; Cutter 2008; Cutter et al. 2008).

Sex determination in Caenorhabditis is an XO type chromosomal system, with females/hermaphrodites having two copies of the $X$ chromosome and males having a single $X$ chromosome (Herman 2005). Laboratory populations of C. elegans kept under constant conditions in which the frequency of males is initially elevated above the background typically lose males, until the frequency of males equilibrates at the frequency of non-disjunction of the $\mathrm{X}$ (Stewart and Phillips 2002; Teotonio et al. 2006). The frequency of males varies among strains (Hodgkin and Doniach 1997; Teotonio et al. 2006) and depends on environmental conditions, averaging about $0.1 \%$ under standard husbandry conditions in the N2 strain. However, laboratory populations exposed to variable or directional selection can maintain males at significantly higher frequencies (Anderson et al. 2010; Teotonio et al. 2012), 
consistent with the idea that recombination facilitates adaptive evolution.

In an androdioecioius population, selection on male function is (i) necessarily weaker than selection on hermaphrodite function and (ii) weaker than selection on male or female function in a dioecious population, because in the absence of males (or females) a dioecious population immediately goes extinct whereas an androdioecious population persists even in the complete absence of males. Moreover, although selection typically favors an equal sexratio in a randomly mating dioecious population (Fisher 1930), that is not generally true in a partially selfing population (Charnov 1982).

Importantly, the rarer males become, the less efficient is selection against genes with male-specific effects on fitness. For example, if males represent $0.1 \%$ of the population, as in a typical C. elegans lab population, an autosomal gene with effects only on male fitness will find itself in a maleand thus under selection $-0.1 \%$ of the time and in a hermaphrodite-and thus free from selection $-99.9 \%$ of the time. If the selection coefficient on that gene is $10 \%$ in males and 0 in females, the average selection coefficient at the gene will be $0.01 \%$. The effective population size, $N_{e}$, of C. elegans is thought to be on the order of $10^{4}$ (Andersen et al. 2012), so an allele with those sex-specific selection coefficients will have an average selection coefficient of approximately $1 / N_{e}$, roughly the boundary of effective neutrality (Ohta 1973). Thus, the rarer males become, the stronger selection must be on male function to overcome random genetic drift.

These features of selection in androdioecious populations lead to a chicken-and-egg question with respect to the rarity of males in androdioecious Caenorhabditis: are males rare because the sex-ratio is near an evolutionary optimum, or are males on their way out, doomed to ultimately succumb to the ravages of deleterious mutation? Or perhaps both. A theoretical investigation concluded that an obligately selfing population of $C$. elegans would go extinct by mutational meltdown after only a few thousand generations (Loewe and Cutter 2008), although those calculations are based on an estimate of the genome-wide deleterious mutation rate that is probably inflated by a few fold (see below). The absence of obligate-selfing species in the genus, and the typically short evolutionary lives of obligate selfing taxa more generally, suggest that obligate selfing is an evolutionary dead-end (Igic and Busch 2013).

A quantitative assessment of the long-term stability of androdioecy in $C$. elegans requires an estimate of the distribution of effects of mutations affecting male fitness, both with respect to mutations that render males non-viable or sterile, and the effects on the ability of males to mate and for male sperm to compete with hermaphrodite sperm. Several elements of male fitness have been shown to be genetically variable among wild isolates of $C$. elegans (Murray et al. 2011; Teotonio et al. 2006). Moreover, an initially isogenic population of $C$. elegans evolved increased male mating ability over 100 generations of adaptation to laboratory conditions (Teotonio et al. 2012), which indicates both significant input of mutational variance for male fitness and that periodic outcrossing is adaptive under the experimental conditions.

Unfortunately, the distribution of fitness effects (DFE) on individual traits is very difficult to quantify reliably (Charlesworth 2015). More tractable measures of the vulnerability of a trait to the cumulative effects of mutation are (i) the rate of change of the trait mean due to the accumulation of spontaneous mutations (the "mutational bias", $\Delta \mathrm{M}$ ) and (ii) the rate of increase in genetic variance (the "mutational variance", VM). These quantities can be used to quantify the relative mutability of traits and populations. If an estimate of the genome-wide mutation rate $\mu_{G}$ is available, $\Delta \mathrm{M} / \mu_{G}$ gives the average mutational effect on the trait.

Here we report results of a study designed to estimate the cumulative effects of spontaneous mutations on male-male competitive fitness in a set of $C$. elegans mutation accumulation (MA) lines which were propagated by single hermaphrodite descent for approximately 250 generations. In this context, male function is a truly neutral trait, since chromosomes were (almost) never passed through males. Cumulative effects of mutations on many hermaphrodite traits have been previously reported for these and other $C$. elegans MA lines (summarized in Davies et al. (2016)), providing a robust baseline against which to compare male mutational properties. In addition, we report new results on the cumulative mutational effects on hermaphroditehermaphrodite competitive fitness.

The results will shed light on two questions of interest. First, the frequency of MA lines for which fertile males can be obtained provides a rough upper bound on the class of mutations resulting in "zero male fitness" (ZMF); this class comprises male-specific lethal and male-sterile mutations. There are surprisingly few published estimates of the rate of mutation to alleles of zero male fitness. Mukai et al. (1972) reported the frequency of matings of Drosophila melanogaster MA lines resulting in sterility was "below $2 \%$ ". Willis (1999) reported that a large fraction of inbreeding depression in Mimulus gutatus $(\sim 30 \%)$ could be attributed to male-sterile mutations, but actual rates could not be quantified. Calculations from data in Fig. 1 of Sharp and Agrawal (2013) using the method we apply to our own data suggest a male-sterile mutation rate in D. melanogaster of about $0.16 \%$ per genome/generation, about a tenth the rate of lethal recessives (Drake et al. 1998). Application of our method to data from a different MA experiment with the N2 strain of C. elegans (Vassilieva et al. 2000) gives an 
upper bound on the ZMF mutation rate of about $0.03 \%$ / generation.

Second, male components of fitness may be particularly vulnerable to the effects of deleterious mutations, for two reasons. First, small differences in performance may be magnified into large differences in mating success, with the winner mating and the loser not mating. That scenario underpins Bateman's principle, the observation that the variance in reproductive success is usually greater in males than in females (Bateman 1948). Also, the "genic capture" hypothesis (Rowe and Houle 1996) predicts that sexual selection acts at least indirectly on male physiological condition, in which case the mutational target of male fitness is potentially very large. Given the infrequency of outcrossing in C. elegans, it is arguable whether sexual selection is important to the evolution of the species. However, given that $(i)$ outcrossing is the ancestral state in the genus, (ii) the three known androdioecious Caenorhabitis species all represent tip clades in the phylogeny of the genus (Kiontke et al. 2011), and (iii) the basic biology of mating and fertilization appears at least superficially similar throughout the genus (e.g., the use of indole ascarosides as pheromones; Dong et al. 2016), it seems reasonable that the cumulative mutational effects on male fitness in C. elegans would approximate those in dioecious species.

To date, the only published estimates of the cumulative effects of spontaneous mutations on male fitness in any animal come from Drosophila melanogaster (Mallet et al. 2011; Mallet et al. 2012; Sharp and Agrawal 2013), in which effects on male fitness are generally somewhat greater than those on female fitness.

\section{Materials and methods}

\section{Mutation accumulation (MA)}

Details of the MA experiment have been reported elsewhere (Baer et al. 2005). Briefly, 100 replicate lines were initiated from a nearly isogenic population of the $\mathrm{N} 2$ strain of $C$. elegans and maintained by serial transfer of a single immature hermaphrodite every generation for approximately 250 generations, at which time ("G250") the MA lines were cryopreserved. The common ancestor of the MA lines ("G0") was cryopreserved at the outset of the experiment.

\section{Recovery of males from MA lines}

In 2015, the 60 remaining G250 MA lines were thawed and replicate populations collected on standard $60 \mathrm{~mm}$ NGM agar plates. For lines in which males were not initially present, we attempted to generate males using a standard heat shock protocol to induce non-disjunction of the $\mathrm{X}(\mathrm{He}$ 2011). If males were obtained but pairings with hermaphrodites failed to produce male progeny, after three heat shock attempts the MA line was characterized as producing sterile males. If no males were obtained after three heat shock attempts, the MA line was characterized as incapable of producing males. Once fertile males were obtained, a single male was paired with three young L4-stage hermaphrodites on a $35 \mathrm{~mm}$ NGM agar plate seeded with OP50 strain E. coli, and the progeny split into two $100 \mathrm{~mm}$ NGM agar plates seeded with OP50, grown until food was just exhausted, and cryopreserved. A set of 46 malesegregating G0 "pseudolines" was constructed in the same way.

\section{Male competitive fitness assay}

Male-male competitive fitness was assayed by pairing a focal male (G0 ancestor or MA) with a marked competitor male of the ST2 strain (homozygous for the dominant ncls2 pH20::GFP reporter allele on an N2 genetic background) and a male-sterile hermaphrodite homozygous for a recessive null allele at the fog-2 locus [fog-2(q71)]. fog-2 is a recessive mutation that destroys spermatogenesis in hermaphrodites, thereby rendering hermaphrodites functionally female (Schedl and Kimble 1988). To minimize segregating variance in the maternal stock, we backcrossed the fog-2 (q71) mutant allele into the ancestral N2 genetic background for ten generations prior to initiating the competitor population from a cross of a ST2 male with a fog-2 female. We refer to this assay as an assay of "male-male competitive fitness", but the assay also encompasses female choice, and therefore does not measure only male-male competition in the strict sense.

The assay was performed in two blocks, under the same conditions as the MA phase of the experiment, with the exception that the assay plates were $40 \%$ agarose (NGMA) rather than $100 \%$ agar, to prevent worms from burying. Each MA line was included in each block. Assay blocks were initiated by thawing MA lines and G0 pseudolines, followed by one generation of male recovery in ten replicate $35 \mathrm{~mm}$ plates. Each replicate plate contained two or three males and three young hermaphrodites. All lines were thawed and replicated on the same day. Replicate plates were assigned random numbers and were subsequently handled in order by random number. On the third day after the replicate plates were initiated, competition assay plates were initiated by transfer of a single young focal male from each replicate plate and a single similarly-staged competitor male from a stock plate. The two males were allowed to acclimate to the plate for 1 day, at which time a female was introduced to the plate at a location approximately intermediate between the two males. Two days after the 
introduction of the female, the three adult worms were removed from the plate and their progeny allowed to grow for another 2 days. In Block 1, after the two-day growth period, plates were stored at $4{ }^{\circ} \mathrm{C}$ for 4 days prior to counting. In Block 2 , worms were counted directly after the two-day growth period without refrigeration.

Worms were counted and categorized as fluorescent or wild-type with the aid of a Union Biometrica BioSorter ${ }^{\mathrm{TM}}$ large-particle flow cytometer (aka, a "worm sorter") equipped with the LP Sampler ${ }^{\mathrm{TM}}$ microtiter plate sampler. The detailed counting protocol is given in Supplementary Appendix A1.

\section{Hermaphrodite competitive fitness assay}

Competitive fitness of hermaphrodites was assayed in two blocks beginning in May, 2005. At the outset of each block, the cryopreserved G0 ancestor of the MA lines was thawed and 20 replicate populations initiated from a single L3/L4 stage worm placed on a standard $60 \mathrm{~mm}$ NGM agar plate seeded with $100 \mu \mathrm{l}$ of an overnight culture of the OP50 strain of E. coli. These populations are referred to as "pseudolines" and designated the P0 generation. Seven L3/L4 stage offspring from each pseudoline were transferred singly to new plates, designated the P1 generation. From this point on, pseudolines were treated identically to the MA lines. G250 MA lines were thawed and seven revived L3/L4 stage worms from each line were placed individually on standard $60 \mathrm{~mm}$ NGM plates, labeled P1. All P1 plates were assigned a unique random number and all subsequent experimental manipulations were performed in sequence by random number. Replicates were maintained for two more generations (P2-P3) by transfer of a single L3/L4 stage offspring at four-day intervals to control for parental and grandparental effects. At the same time, we thawed a replicate of the GFP-marked competitor strain ST2 and made several large replicate populations by transferring a chunk from the initial plate to a new $100 \mathrm{~mm}$ plate.

On the second day after the P3 worm began reproduction, a competition plate for each replicate was set up by transferring a single L1-stage larva from the P3 plate and a single L1-stage ST2 competitor onto a $60 \mathrm{~mm}$ NGM agar plate seeded with $100 \mu \mathrm{l}$ of the HB101 strain of E. coli and supplemented with nystatin to retard fungal contamination. Competition plates were incubated at $20^{\circ} \mathrm{C}$ for 8 days, at which point food was exhausted. Worms were washed from competition plates in cold M9 buffer, settled on ice and $100 \mu \mathrm{l}$ of the settled worms transferred into a drop of glycerol on the lid of an empty $60 \mathrm{~mm}$ agar dish and the bottom of the empty dish pressed into the lid. The glycerol immobilizes the worms and pressing them between halves of the plate puts them into the same focal plane. We took two pictures of each plate at $40 \mathrm{X}$ magnification through a
Leica MZ75 dissecting microscope fitted with a $100 \mathrm{~W}$ mercury arc lamp and epifluorescence GFP filter cube (470/ $40 \mathrm{~nm}$ excitation filter, $525 / 50 \mathrm{~nm}$ emission filter) using a Leica DFC280 camera connected to a computer running the Leica IM50 software (Leica Microsystems Imaging Solutions Ltd). The first picture used the arc lamp and GFP filter cube (the "green" image) and the second, taken immediately afterwards, used transmitted white light (the "white" image). All worms are visible in the white image, whereas wild-type (non-GFP) worms appear only faintly in the green image (Supplementary Figure S1). The difference between the number of worms in a white image and in the matching green image is the number of focal worms in the sample.

Images were imported into ImageJ software (http://rsb. info.nih.gov/ij/) and worms were counted as follows. If there appeared to be fewer than 200 worms visible in a white image, we first counted every worm in the white image and then each worm visible in the accompanying green image. If there appeared to be $>200$ worms in the white image we drew a rectangle around approximately 200 worms and counted them. We then pasted the same rectangle in the green image and counted the worms visible within the rectangle.

\section{Data analysis}

i) Measures of competitive fitness-Competitive fitness has two components: (1) did the focal individual reproduce at all? If not, relative fitness is zero regardless of the number of offspring of the competitor, and (2) given that the focal individual did reproduce, what fraction of the offspring belong to the focal individual? These considerations apply both to male-male and hermaphrodite-hermaphrodite competitive fitness. Given that a focal individual did reproduce, the ratio $p /(1-p)$ is related to competitive fitness by the relationship

$\frac{p_{t}}{q_{t}}=\frac{p_{0}}{q_{0}}\left(\frac{W_{f o c}}{W_{C}}\right)^{t}$

(Barton et al. 2007, Equation 17.2), where $t$ represents the number of generations in the fitness assay (NOT the number of MA generations), $p_{0}$ is the frequency of the focal type (G0 or control) at the beginning of the assay, $p_{t}$ is the frequency of the focal type (G0 control or MA) at the conclusion of the assay, $q=1-p, W_{f o c}$ is the absolute fitness of the focal type, and $W_{C}$ is the absolute fitness of the competitor. Each trial was started with one focal worm and one competitor, so the ratio $\frac{p_{0}}{q_{0}}=1$. We refer to the ratio $p /(1-p)$ as the "competitive index", $C I$ (Shabalina et al. 1997). CI provides a measure of fitness of the focal type relative to the competitor, raised to the power $t$. All analyses of $C I$ were performed on natural log-transformed data. 
The proportion of offspring of a cross that survive to be counted is a function of the dominance of mutant alleles. Vassilieva et al. (2000) estimated the coefficient of dominance of mutations affecting survivorship in $\mathrm{N} 2$ to be 0.05 , i.e., mutations that influence survivorship are nearly completely recessive. Therefore, any reduction in fitness of males from MA lines relative to the wild-type must be the property of the male itself rather than of offspring survivorship. From data given in Supplementary Table S2, we estimate the reduction in homozygous offspring survival to be about $0.048 \%$ /generation, or about $12 \%$ over the course of 250 generations of MA. If the coefficient of dominance is 0.05 , mutant heterozygotes will have a probability of surviving of $1-h s=1-(0.05)(0.12)=99.4 \%$ that of the homozygote. If the coefficient of dominance is as large as 0.33 (the upper $95 \%$ confidence limit estimated by Vassilieva et al. (2000)), the reduction in heterozygous survivorship will be about $4 \%$.

ii) Probability of reproduction, $\underline{\pi}$-Probability of reproduction is a binary trait. If a focal worm reproduced the replicate is scored as a success ("event $=1$ "); if the focal worm did not reproduce it is scored as a failure ("event = 0"). Data were analyzed by Generalized Linear Mixed Model (GLMM) with estimation by Residual Subjectspecific Pseudolikelihood (RSPL) as implemented in the GLIMMIX procedure of SAS v.9.4 with a logit link function and a random residual. Treatment (MA vs. Control) is a fixed effect and Line and Replicate (nested within Line) are random effects. Block is a random effect in principle. However, pseudolikelihoods are not appropriate criteria for model selection (e.g., by AIC; SAS/Stat User's Guide (2013)), so rather than include or exclude variance components including block on the basis of estimates for which there is little power (because $n=2$ ), we chose to model block as a fixed effect for this analysis. It is common in the analysis of MA fitness assays to treat block as a fixed effect when the number of blocks is small (e.g., Houle et al. 1994; Shaw et al. 2000).

Each line (MA and G0 pseudoline) was assayed for probability of reproducing, $\pi_{M}$, in each of the two assay blocks. The full model is written as:

$\pi_{i j k l}=\mu+t_{k}+b_{j}+c_{j k}+l_{l \mid j k}+\varepsilon_{i l j k}$

where $\pi_{i j k l}$ is a binary variate scored as 1 if the focal worm produced at least two offspring and 0 if it did not, $\mu$ is the overall mean, $t_{k}$ is the fixed effect of treatment $k$ (G0 or MA), $b_{j}$ is the fixed effect of block $j, c_{j k}$ is the fixed effect of the treatment by block interaction, $l_{l \mid j k}$ is the random effect of line (or pseudoline) $l$, conditioned on block and treatment, and $\varepsilon_{i l j k}$ is the random residual, conditioned on block and treatment. Random effects were estimated separately for each block/treatment combination by means of the GROUP option in the RANDOM statement of the
GLIMMIX procedure. Significance of fixed effects was determined by F-test of Type III sums of squares.

Hermaphrodite probability of reproduction, $\pi_{H}$, was modelled similarly, with the exception that each line (or pseudoline) was represented in only one of the two assay blocks, so line is nested within block. The distribution of $\pi_{H}$ was strongly left-skewed, so means and standard errors were calculated by an empirical bootstrap procedure (Baer et al. 2006; Efron and Tibshirani 1993). Resampled datasets were constructed by resampling lines within blocks, followed by estimation of means and variance components from the GLMM described above.

\section{Competitive Index (CI)}

(i) Males. Given that both male worms-the focal worm and the competitor-sired at least $2 \%$ of the offspring on the competition plate, we analyzed false-positive corrected male-male $C I\left(C I_{M}\right)$ using a standard general linear model (GLM) as implemented in the MIXED procedure of SAS v. 9.4. The correction for false positives is explained in Supplementary Appendix A1. Studentized residuals of natural log-transformed data were scrutinized for outliers by eye against a Q-Q plot. After removal of three outliers $(n=$ 671), the data were initially fit to the linear model:

$y_{i j k l}=\mu+t_{k}+b_{j \mid k}+c_{j k}+l_{l \mid j k}+\varepsilon_{i l \mid j k}$

where $y_{i j k l}$ is the $\log (C I)$ of the individual replicate and the independent variables are defined as in the previous section. Block and Line-by-block interaction were modelled as random effects in this analysis. Variance components of random effects were estimated by restricted maximum likelihood (REML). The among-block component of variance was estimated separately for each treatment and the among-line and among-replicate (nested within line) components were estimated separately for each treatment/block combination by means of the GROUP option in the RANDOM or REPEATED statement of the MIXED procedure (Fry 2004).

We first analyzed the full model above, then sequentially simplified the model by first pooling the random effects across grouping levels (e.g., estimating a single among-line variance rather than estimating it separately for each block) and then removing the effect entirely. The model with the smallest corrected AIC (AICc) was chosen as the best model, and significance of the fixed effect of treatment (MA or G0) in that model was determined by F-test of Type III sums of squares, with degrees of freedom determined by the Kenward-Roger method (Kenward and Roger 1997). If two models had equal AICc, the simpler model was chosen as the best model. AICc's of the models tested are given in Supplementary Table S1. In addition, we calculated empirical bootstrap estimates of the mean and standard error 
of $C I_{M}$, resampling over lines within blocks followed by estimation of means and variance components from the GLM described above.

Hermaphrodite $C I, C I_{H}$ was modelled analogously to $C I_{M}$, with the exception that each line (or pseudoline) was represented in only one of the two assay blocks, therefore line is nested within block and there is no line-by-block interaction term. Outliers were identified as for males; five outliers $(n=727)$ were removed prior to further analysis.

\section{Mutational bias}

The mutational bias is the per-generation rate of change in the trait mean. The slope of the regression of trait mean on generation of MA is often designated $R_{M}$ (Vassilieva and Lynch 1999); the per-generation change scaled as a fraction of the ancestral (G0) trait mean is often referred to as $\Delta M_{z}=\frac{\bar{z}_{M A}-\bar{z}_{0}}{t \bar{z}_{0}}$, where $\bar{z}_{M A}$ and $\bar{z}_{0}$ represent the MA and ancestral trait means and $t$ is the number of generations of MA. For $\pi_{M}$ and $\pi_{H}$, MA and G0 means were estimated by least squares, given the general linear mixed model, and $\Delta \mathrm{M}_{\pi}$ calculated directly from the least-squares means. $C I$ is on a logarithmic scale so mean-standardization of the data is not appropriate because $C I$ can be negative or positive. For $C I, R_{M, C I}=\frac{\bar{z}_{M A}-\bar{z}_{0}}{t}$ and the mutational bias represents the per-generation change in competitive fitness of the focal genotype relative to the competitor strain. $R_{M, C I}$ can be related to the per-generation mutational change in relative fitness per se, $\Delta \mathrm{M}_{w}$, from Eq. 1, as explained below in the Results.

\section{Mutational variance $\left(\mathrm{V}_{\mathrm{M}}\right)$}

The per-generation increase in genetic variance resulting from new mutations, $V_{M}$, is equal to the product of the pergenome, per-generation mutation rate $\left(\mu_{G}\right)$ and the square of the average effect of a mutation on the trait of interest, $\alpha$, i.e., $V_{M}=\mu_{G} \alpha^{2}$ (Barton 1990). In this experiment, MA lines are assumed to be homozygous, in which case $V_{M}$ is equal to half the increase in the among-line component of phenotypic variance, divided by the number of generations of MA, i.e, $V_{M}=\Delta V_{L}=\frac{V_{L, M A}-V_{L, G 0}}{2 t}$, where $V_{L, M A}$ is the variance among MA lines, $V_{L, G 0}$ is the variance among the G0 pseudolines, and $t$ is the number of generations of MA (Lynch and Walsh 1998, p. 330). For all traits ( $\pi$ and $C I$ ), $\mathrm{VL}$ is the among-line component of variance of the treatment group (MA or G0) extracted from the relevant GLM or GLMM.

$V_{M}$ is typically scaled either relative to the residual (environmental) variance, $V_{E}$ or to the square of the trait mean, $\bar{z}$. The ratio $V_{M} / V_{E}$ is the mutational heritability $\left(h_{M}^{2}\right)$, and the ratio $\frac{V M}{\bar{z}^{2}}$ is sometimes called the mutational evolvability $\left(I_{M}\right)$ and is equivalent to the squared mutational coefficient of variation (Houle et al. 1996). Usually, $V_{M}$ is scaled relative to the ancestral (G0) mean, but if the mean changes substantially over the course of MA (i.e., $\Delta \mathrm{M} \neq 0$ ), it is more meaningful to scale each group (G0 and MA) by its own mean (Baer et al. 2005). Scaling by the group means is nearly equivalent to calculating $\Delta V_{L}$ from $\log$ transformed data (Fry and Heinsohn 2002). CI is on a logarithmic scale and cannot be mean-standardized. $\Delta V_{L}$ for $C I_{M}$ and for $\pi_{M}$ and $\pi_{H}$ is not significantly different from 0 (see Results), so scaling is irrelevant.

\section{Results}

\section{Rate of mutations with zero male fitness}

Of the 60 of the original 100 MA lines remaining in 2014, we were able to obtain fertile males from 53. We assume that the seven lines for which we were unable to obtain fertile males carry at least one mutation that leads to Zero Male Fitness (ZMF, i.e. inviable or sterile), and that these mutations follow a Poisson distribution-analogous to lethal equivalents (Morton et al. 1956). With those assumptions, the expected proportion of lines with no mutations $\left(p_{0}\right.$, i.e., the number of lines that produced fertile males) is: $p_{0}=e^{-m}$, where $m$ is the expected number of mutations carried by a line (Luria and Delbrück 1943). The expected number of mutations $m=\mu t$, where $\mu$ is the pergeneration rate of ZMF mutations and $t$ is the number of generations of MA. Thus, $(53 / 60)=e^{-250 \mu}$, so $\mu_{Z M F} \approx 5 \times$ $10^{-4}$ /generation, about half the lower-bound estimate on the frequency of males. If the ZMF mutation rate is greater than the frequency of males, the average chromosome will take a male-sterilizing hit before the next time it winds up in a male, leading to the loss of males by "error catastrophe" (Eigen 1971). The same calculation from data reported in Vassilieva et al. (2000) gives an estimate of $\mu_{Z M F} \approx 3 \times 10^{-4} /$ generation.

\section{Male-male competitive fitness}

(i) Probability of mating $\left(\pi_{M}\right)$. After 250 generations of completely relaxed selection, MA males are significantly less likely to successfully mate under competitive conditions than are their unmutated $\mathrm{G} 0$ ancestors $\left(\mathrm{F}_{1,131.8}=\right.$ 12.51, $P<0.0001)$. Averaged over the two blocks, the probability that a G0 male mated successfully (defined as an estimated frequency of offspring sired $>2 \%$ ) was $\sim 90 \%$ $\left(\bar{\pi}_{M}=0.912 \pm 0.019\right)$ whereas the probability that a MA male mated significantly declined to $\sim 75 \%$ $\left(\bar{\pi}_{M}=0.757 \pm 0.023\right)$. Scaled relative to the G0 mean, the probability of a male successfully mating under the assay conditions decreased by about $0.06 \%$ per generation $\left(\Delta \mathrm{M}_{\pi}\right.$ 
$=-0.622+0.159 \times 10^{-3}$ /generation; Table 1 ; distributions of line means are shown in Supplementary Figure S2).

In neither block did $V_{M}$ of $\pi_{M}$ differ significantly from 0 . In the first block, the RSPL estimate of $\mathrm{V}_{\mathrm{L}}$ among G0 pseudolines was greater than $\mathrm{V}_{\mathrm{L}}$ among MA lines; in the second block $\mathrm{V}_{\mathrm{L}}$ was greater in the MA lines but the difference was not significant (Table 2).

(ii) Competitive Index $\left(C I_{M}\right)$. When the estimated frequency of offspring sired by the focal male was at least $2 \%$, MA males sired a smaller fraction of offspring than did their unmutated $\mathrm{G} 0$ ancestors $\left[\log \left(C I_{M, G 0}\right)=-0.145+0.074\right.$; $\log \left(C I_{M, M A}\right)=-0.461+0.095$; standard errors represented by the standard deviation of the empirical bootstrap distribution]. The best-fit linear model includes a separate among-block component of variance for each treatment (Supplementary Table S1), and under that model the change in the trait mean is not significantly different from zero $\left(\mathrm{F}_{1,1.38}=1.67, P>0.37\right)$. However, when the among-block variance is pooled over the two treatments, the change in the mean $C I_{M}$ becomes significant $\left(\mathrm{F}_{1,603}=5.57, P<0.02\right)$. The lack of significance in the best-fit model potentially represents Type II error resulting from having to estimate a variance component with $n=2$. To test that possibility, we estimated the change in the trait mean from the mean of 1000 bootstrap replicates, resulting in an empirical $P<$ 0.007 . Averaged over the two blocks, $\log \left(C I_{M}\right)$ declined by slightly more than $0.1 \%$ per generation $\left(R_{M, C l, M}=-1.26 \pm\right.$ $0.48 \times 10^{-3}$ /generation; Table 1 ; distributions of line means are shown in Supplementary Figure S3).

As noted, $C I$ cannot be directly mean-standardized. However, $C I$ is related to relative fitness by Eq. (1) above. $\Delta \mathrm{M}_{W}$ can be calculated from $\frac{p_{t}}{q_{t}}=\frac{p_{0}}{q_{0}}\left(\frac{W_{f o c}}{W_{C}}\right)^{t}$, where $C I=\left(\frac{p_{t}}{q_{t}}\right), p_{0}=q_{0}=0.5$, and $t=1$, thus $C I=\left(\frac{W_{f o c}}{W_{C}}\right)$. The ratio of the fitnesses relative to the competitor (designated by a capital $W),\left(\frac{W_{M A}}{W_{C}}\right)\left(\frac{W_{G 0}}{W_{C}}\right)^{-1}$ gives the fitness of the MA lines relative to that of the G0 ancestor (designated by a lower-case $w),\left(\frac{w_{M A}}{w_{G 0}}\right)$. The ratio $\left(\frac{W_{G 0}}{W_{C}}\right)=\exp (-0.145)=$ 0.865 and $\left(\frac{W_{M A}}{W_{C}}\right)=\exp (-0.461)=0.631$, so $\left(\frac{w_{M A}}{w_{G O}}\right) \approx 0.73$. Thus, male competitive fitness relative to the ST2 competitor declined by about $27 \%$ over the course of 250 generations of MA, or by about $-1.04 \times 10^{-3} /$ generation when scaled as a fraction of the G0 mean. That calculation does not account for the presumably small reduction in offspring number resulting from heterozygous effects on offspring survival.

The REML estimate of $\mathrm{V}_{\mathrm{L}}$ for $\log \left(C I_{M}\right)$ for both $\mathrm{G} 0$ pseudolines and MA lines is zero in each block (Table 2), and the distributions of line means are similar in the two groups (Supplementary Figure S3). Taken at face value, a change in the mean coupled with no change in the amongline variance implies that each line changed at the same rate, or at least at rates that were indistinguishable. A more plausible explanation is that the true genetic variance is small relative to the environmental variance (which includes experimental error) and the sample sizes employed here were not large enough to provide power to detect small differences. In the male fitness assay, each line was initially replicated tenfold, five replicates per block. In the hermaphrodite competitive fitness assay, in which $\Delta \mathrm{V}_{\mathrm{L}}$ for $\log$ $\left(C I_{H}\right)$ is highly significant $(P<0.0001$; see next section), each line was replicated sevenfold, but in only one of the two blocks.

\section{Hermaphrodite-hermaphrodite competitive fitness}

(i) Probability of reproducing $\left(\pi_{H}\right)$. The probability of a hermaphrodite reproducing was high $(>98 \%$ for both G0 and MA), and changed little over 250 generations of MA $\left(\Delta \mathrm{M}=-1.9 \times 10^{-5}\right.$ /generation; Table 1 ; distributions of line means are shown in Supplementary Figure S2). The mutational heritability is large $\left(h_{M}^{2} \approx 0.02\right.$; Table 2$)$, but the
Table 1 Evolution of trait means, averaged across blocks; standard errors in parentheses

\begin{tabular}{|c|c|c|c|c|}
\hline & $\pi_{M}$ & $\pi_{H}$ & $\log \left(C I_{M}\right)$ & $\log \left(C I_{H}\right)$ \\
\hline G0 & $0.912(0.016)$ & $0.987(0.008)$ & $-0.145(0.074)$ & $0.862(0.331)$ \\
\hline MA & $0.757(0.023)$ & $0.982(0.005)$ & $-0.461(0.095)$ & $0.228(0.338)$ \\
\hline$R_{M}\left(\mathrm{x} 10^{3} /\right.$ gen $)$ & $-0.622(0.159)$ & $-0.0192(0.050)$ & $-1.26(0.48)$ & $-2.54(0.49)$ \\
\hline$\Delta \mathrm{M}\left(\mathrm{x} 10^{3} /\right.$ gen $)$ & -0.682 & -0.0195 & $-1.04^{\dagger}$ & $-1.09^{*}, \dagger$ \\
\hline
\end{tabular}

Sample sizes are given in Table 2. Abbreviations are: $\pi_{M}$, probability of male reproduction; $\pi_{H}$, probability of hermaphrodite reproduction; $C I_{M}$, male competitive index; $C I_{H}$, hermaphrodite competitive index; $R_{M}$, slope of the regression of the trait mean on generation of MA; $\Delta \mathrm{M}$, the per-generation change in the trait mean scaled as a fraction of the G0 mean. Values of $R_{M}$ significantly different from zero $(P<0.05)$ are shown in bold type. See Methods for details of calculations. $\Delta \mathrm{M}$ of $C I$ is calculated for back-transformed data.

*Assumes $t=2$ generations of reproduction

${ }^{\dagger} \Delta \mathrm{M}$ calculated for $C I$, not $\log (C I)$ 
Table 2 Variances. Standard errors in parentheses

\begin{tabular}{|c|c|c|c|c|c|}
\hline Variance Component & Block & $\begin{array}{l}\pi_{M} \\
n_{M A, B l k I}=46 / 51 \\
n_{M A, B l k 2}=53 / 53\end{array}$ & $\begin{array}{l}\pi_{H} \\
n_{M A, B l k 1}=41 \\
n_{M A, B l k 2}=39\end{array}$ & $\begin{array}{l}\log \left(C I_{M}\right) \\
n_{M A, B l k 1}=46 / 53 \\
n_{M A, B l k 2}=53 / 53\end{array}$ & $\begin{array}{l}\log \left(C I_{H}\right) \\
n_{M A, B l k 1}=41 \\
n_{M A, B l k 2}=39\end{array}$ \\
\hline \multirow[t]{3}{*}{$\mathrm{V}_{\mathrm{L}}(\mathrm{G} 0)$} & 1 & $2.662(0.953)$ & 0 & & 0 \\
\hline & 2 & $0.705(0.319)$ & 0 & & 0 \\
\hline & Ave & $0.574(0.288)$ & 0 & 0 & 0 \\
\hline \multirow[t]{3}{*}{$\mathrm{V} L(\mathrm{MA})$} & 1 & $0.256(0.406)$ & $3.547(1.213)$ & & $0.743(0.212)$ \\
\hline & 2 & $0.412(0.304)$ & $2.546(0.952)$ & & $0.343(0.147)$ \\
\hline & Ave & $0.266(0.187)$ & $3.052^{1}(0,4.299)$ & 0 & $0.571(0.128)$ \\
\hline $\mathrm{V}_{\mathrm{M}}\left(\mathrm{x} \quad 10^{3}\right)$ & Ave & 0 & $6.104^{1}(0,8.598)$ & 0 & $1.142(0.256)$ \\
\hline \multirow[t]{3}{*}{$\mathrm{V}_{\mathrm{E}}(\mathrm{G} 0)$} & 1 & $0.328(0.037)$ & $1.008(0.130)$ & & \\
\hline & 2 & $0.823(0.093)$ & $1.007(0.120)$ & & \\
\hline & Ave & $0.644(0.052)$ & $1.008(0.088)$ & $2.211^{2}(0.173)$ & $0.822^{2}(0.068)$ \\
\hline \multirow[t]{3}{*}{$\mathrm{V}_{\mathrm{E}}(\mathrm{MA})$} & 1 & $0.900(0.105)$ & $0.261(0.025)$ & & \\
\hline & 2 & $0.903(0.099)$ & $0.341(0.030)$ & & \\
\hline & Ave & $0.902(0.072)$ & $0.301(0.020)$ & $3.408^{2}(0.298)$ & $1.796^{2}(0.136)$ \\
\hline$h_{M}^{2}\left(\mathrm{x} 10^{3}\right)$ & Ave & 0 & 20.0 & 0 & 0.841 \\
\hline
\end{tabular}

Abbreviations of variance components are: $\mathrm{V}_{\mathrm{L}}$, among-line component of variance; $\mathrm{V}_{\mathrm{M}}$, mutational variance; $\mathrm{V}_{\mathrm{E}}$, residual (=environmental) component of variance; $h_{M}^{2}$, mutational heritability $\left(=\mathrm{V}_{\mathrm{M}} / \mathrm{V}_{\mathrm{E}}\right.$, where $\mathrm{V}_{\mathrm{E}}$ is calculated from the MA lines). Other abbreviations are as in Table 1. See Methods for details of calculations. For male traits, $n_{M A, B l k}$. is the fraction of the $53 \mathrm{MA}$ lines that were included in that block. For hermaphrodite traits, each block had a different set of MA lines, out of 80 total lines. $\mathrm{V}_{\mathrm{M}}$ significantly greater than $0(P<$ 0.05 ) shown in bold type.

${ }^{1} 95 \%$ bootstrap confidence interval in parentheses

${ }^{2}$ Best-fit model includes residual variances for each treatment (G0, MA) pooled over blocks distribution of $\pi_{H}$ among lines is highly left-skewed (median $\pi_{H}=1$; Supplementary Figure S2) so the point estimate of $h_{M}^{2}$ is probably not very meaningful.

It is likely that some GFP-marked competitors were misidentified as focal types (non-GFP) in our image analysis, which could potentially inflate the apparent probability of reproduction. However, these values of $\pi_{H}$ are nearly identical to the probability of reproduction of hermaphrodites in a different experiment in which hermaphrodites of the same set of MA lines were allowed to reproduce in non-competitive conditions $\left(\pi_{H}>97 \%\right.$ for both $\mathrm{G} 0$ and MA; $\Delta \mathrm{M}=-3.3 \times 10^{-5} /$ generation; reanalysis of data in Baer et al. (2006)). Thus, the very high rate of reproduction does not appear to be an experimental artifact.

(ii) Competitive Index $\left(\mathrm{CI}_{H}\right)$-Mean $\mathrm{CI}_{H}$ declined significantly over the course of 250 generations of MA [ $10 \mathrm{~g}$ $\left(C I_{H, G O}\right)=0.862+0.331 ; \quad \log \left(C I_{H, M A}\right)=0.228+0.338 ;$ $\mathrm{F}_{1,169}=26.97, P<0.0001$; Table 1 ; distributions of line means are shown in Supplementary Figure S3]. $R_{M, C I, H}$ calculated from the slope of the regression of $\log \left(C I_{H}\right)$ on generation of $\mathrm{MA}$ is $-2.54+0.49 \times 10^{-3}$ per-generation. From Eq. (1), $\frac{p_{t}}{q_{t}}=\frac{p_{0}}{q_{0}}\left(\frac{W_{\text {foc }}}{W_{C}}\right)^{t}$, where $C I=\left(\frac{p_{t}}{q_{t}}\right), p_{0}=q_{0}=0.5$ and here $t$ is equal to the number of generations of reproduction the population underwent over the course of the eight-day assay. Therefore, $\log (C I)=t \times \log \left(\frac{W_{f o c}}{W_{C}}\right)$, so $[\exp (\log (C I))]^{1 / t}=\left(\frac{W_{f o c}}{W_{C}}\right)$, and the ratio of the fitnesses relative to the competitor, $\left(\frac{W_{M A}}{W_{C}}\right)\left(\frac{W_{G 0}}{W_{C}}\right)^{-1}$ gives the fitness of the MA lines relative to that of the G0 ancestor, $\left(\frac{w_{M A}}{w_{G 0}}\right)$.

We cannot be certain about the exact number of generations of reproduction, except that three is the upper bound (based on the worm's life cycle), and the true number is probably close to two. The basis for that judgment is this: if the average worm produces 200 offspring in its lifetime, after one generation there will be $2 \times 200=400$ worms on the plate and after two generations there will be $400 \times 200$ $=80,000$ worms on the plate (density-dependence notwithstanding); after three generations there will be $80,000 \times 200=16$ million. There were many more than 400 and certainly fewer than 80,000 , so we assume two generations is probably close to the true number of generations.

Assuming that $t=2$, we find $\left(\frac{w_{M A}}{w_{G 0}}\right)=0.728$, or in other words, relative competitive fitness declined by about $27 \%$ over 250 generations of MA. Scaled relative to the G0 ancestor, $\Delta \mathrm{M}_{w} \approx 1.09 \times 10^{-3} /$ generation. If $t$ is closer to 3 , $\left(\frac{w_{M A}}{w_{G 0}}\right) \approx 0.81$ and $\Delta \mathrm{M}_{w} \approx 0.76 \times 10^{-3} /$ generation. 
Averaged over the two blocks, the REML estimate of the among-line variance in $\log \left(C I_{H}\right)$ increased from zero in the G0 ancestor to 0.534 in the MA lines, giving $V_{M}=1.09 \times$ $10^{-3} /$ generation (Table 2; Likelihood Ratio Chi-square $=$ 23.7, df $=2, P<0.0001)$. Scaled as a fraction of $V_{E}$, the mutational heritability $h_{m}^{2}=0.83 \times 10^{-3} /$ generation. By way of comparison, the point-estimate of $h_{m}^{2}$ for noncompetitive fitness in these lines under the same conditions is $\sim 1.29 \times 10^{-3} /$ generation (Baer et al. 2006).

\section{Discussion}

A simple but important finding is the close quantitative agreement between our estimate of the Zero Male Fitness mutation rate and that gleaned from the results of a previous MA experiment on the N2 strain (Vassilieva et al. 2000). Those estimates are subject to several sources of uncertainty, both experimental (e.g., perhaps if we had tried harder, we could have obtained fertile males from the lines that did not produce them) and biological (the distribution of mutational effects). The experimental uncertainty in this case leads to an overestimate of the $\mathrm{ZMF}$ rate. The lethal recessive mutation rate in $\mathrm{N} 2$ has been estimated, from very limited data, to be on the order of $\sim 1 \%$ /generation (Clark et al. 1988; Rosenbluth et al. 1983).

Published estimates from limited data have put the $C$. elegans per-nucleotide mutation rate between about $2 \times 10$ -9 and $10 \times 10^{-9}$ per site per generation (Denver et al. 2009; Denver et al. 2004; Denver et al. 2012), which translates to a genomic mutation rate $\mu_{G}$ between about 0.2 and 1. Results from much more extensive data suggest that $\mu_{G}$ is about 0.33 (CFB, AS, et al., unpublished; sequence data archived in the NCBI Short Read Archive, project number PRJNA395568), which means that each MA line carries about 80 new mutations. If we assume that each genetic death (lethal equivalents) or male dysfunction $(\mathrm{ZMF})$ is due to a single mutation, the fraction of all mutations that are $\mathrm{ZMF}$ is about $0.15 \%$ (i.e., one $\mathrm{ZMF}$ mutation in each of the seven MA lines that failed to produce fertile males). In every hundred genomes there will be $\sim 33$ new mutations, of which about one will be lethal, so the fraction of mutations that are lethal is $1 / 33$, or about $3 \%$. Thus, we infer that the fraction of ZMF mutations is around $5 \%$ of the lethal fraction.

What of the idea that male fitness is more susceptible to the cumulative effects of mutation than hermaphrodite fitness? Fitness is a function of both survival and reproduction, in which case the per-generation decline in relative fitness $\Delta \mathrm{M}_{\mathrm{w}}=1-\left[\left(1-\Delta \mathrm{M}_{\pi}\right)\left(1-\Delta \mathrm{M}_{\mathrm{CI}}\right)\right]$. In the hermaphrodite assay, there was no discernible effect of mutation accumulation on the probability of reproducing $\left(\pi_{H}\right)$, so the decline in relative fitness with MA is encompassed by the
$0.11 \%$ per generation decline in relative competitive fitness $\left(C I_{H}\right)$. In males, both the probability of siring offspring $\left(\pi_{H}\right)$ and the fraction of offspring sired given that the male did mate successfully $\left(C_{H}\right)$ declined. Calculations based on the point estimates of the $\Delta \mathrm{Ms}$ (Table 1) show that, after one generation of MA, hermaphrodite relative fitness will have declined by $\approx 0.11 \%$ and male relative fitness will have declined by $\approx 0.17 \%$. Thus, male-male competitive fitness as measured here is no less sensitive, and perhaps slightly more sensitive to the cumulative deleterious effects of mutation than is hermaphrodite fitness. This result is quantitatively nearly identical to the finding that male Drosophila melanogaster decline in fitness $\sim 1.5 \mathrm{X}$ faster from mutation accumulation than do females (Sharp and Agrawal 2013).

The cumulative effects of selection depend on both the effects of an allele on fitness (the selection coefficient, $s$ ) and the effective population size, $N_{e}$. The average effect of a mutation on fitness, $\bar{s}$, is simply the total decline in fitness divided by the total number of mutations in the genome, i.e., $\bar{s}=\frac{t \Delta M}{t \mu_{G}}=\frac{\Delta M}{\mu_{G}}$. For hermaphrodites, $\Delta \mathrm{M}_{\mathrm{w}}$ is about $10^{-3} /$ generation; if our estimate of $\mu_{G} \approx 0.33$ is correct, $\bar{s} \approx 0.33 \%$. Teotonio et al. (2006) reported analogous (not identical) measures of competitive male mating ability and hermaphrodite competitive fitness for a set of 28 wild isolates. Reanalysis of their hermaphrodite competitive fitness data (generously provided by $\mathrm{H}$. Teótonio) gives an estimate of the among-line variance of $\log \left(C I_{H}\right)$ of about 0.4 ; for homozygous lines the genotypic variance $\mathrm{V}_{\mathrm{G}}=2 \mathrm{~V}_{\mathrm{L}}$ (Falconer 1989, p. 265). Under broadly applicable circumstances, the ratio $\mathrm{V}_{\mathrm{M}} / \mathrm{V}_{\mathrm{G}} \approx \bar{s}$ (Charlesworth 2015; Houle et al. 1996). The ratio of our estimate of $\mathrm{V}_{\mathrm{M}}\left(\approx 10^{-3}\right)$ to the estimate of $\mathrm{V}_{\mathrm{G}}(0.2)$ gives $\bar{s} \approx 0.5 \%$. Given the many sources of uncertainty, it is encouraging that the two different methods of estimating $\bar{s}$ agree to within a factor of two.

For reasons that we elaborate below, inferences with respect to the strength of selection on mutations affecting male fitness are more problematic. Caveats notwithstanding, however, $\Delta \mathrm{M}_{\mathrm{w}}$ for male relative fitness is about $1.7 \times 10^{-3}$, so $\bar{s} \approx 0.5 \%$. $N_{e}$ of $C$. elegans has been estimated from the standing nucleotide diversity as being on the order of $10^{4}$ (Andersen et al. 2012). If a mutant allele is under selection in males but is neutral in hermaphrodites and males represent $1 \%$ of the population, the average selection coefficient on a mutant autosomal allele would be $(0.99)(0)+(0.01)$ $(0.005)=5 \times 10^{-4}$, on the cusp of effective neutrality. Deleterious alleles with selection coefficients $s \approx 1 / N_{e}$ are the most pernicious with respect to population mean fitness (Schultz and Lynch 1997). On the face of it, it appears that males in C. elegans are in peril of mutating their way out of existence.

However, the conclusion that males are in mutational peril is based on several strong assumptions, all of which 
are likely to be violated at least to some degree. First, the calculation is based on the assumption that mutations that affect male fitness have no pleiotropic effects on hermaphrodite fitness. It was a goal of this study to estimate of the mutational correlation between male fitness and hermaphrodite fitness, because those data would illuminate the extent to which intersex pleiotropy ("intralocus conflict" if effects are antagonistic between the sexes; Rice and Chippindale (2001)) is an inherent feature of genomic architecture, without the confounding influence of natural selection. Unfortunately, the lack of significant mutational variance in either of the male fitness traits $\left(\pi_{M}\right.$ and $\left.C I_{M}\right)$ means the estimate of any covariance with those traits is zero. Reanalysis of the wild isolate data of Teotonio et al. (2006) reveals no significant standing genetic correlation of male and hermaphrodite competitive fitness $\left(r_{G}=0.12\right.$, LRT Chi-square $=0.2, \mathrm{df}=1, P>0.65$ ).

The second strong assumption is that male-male competitive fitness faithfully captures the essence of selection acting on males. However, males compete for fitness not only with other males, but also with the hermaphrodite itself, and in fact, head-to-head competition between male C. elegans is probably rare in nature, although it would not have been rare in the outcrossing common ancestor of $C$. elegans. Measuring male-hermaphrodite competitive fitness in our context requires a recessive marker in the hermaphrodite competitor, so that the offspring of a cross can be distinguished from the hermaphrodite's self-progeny. Unfortunately, we were unable to find a recessive marker that has reasonably high fitness when homozygous and can also be reliably scored at sufficiently high throughput to enable a full assay.

Male-male competitive index includes both a behavioral component and a sperm-competition component, which cannot be discriminated with our assay. Both features could potentially affect male-hermaphrodite competitive fitness. A hermaphrodite paired with a male that is a poor mater will presumably sire a larger fraction of offspring prior to exhaustion of its sperm than will a hermaphrodite paired with a good mater. Although we can't be certain, it seems likely that the fraction of males that failed to sire offspring in our head-to-head assay would be greater than the fraction of males that would fail to sire offspring in the absence of a competitor, in which case our estimate of $\pi_{M}$ provides an upwardly-biased estimate of the strength of selection on male mating ability. That inference does not depend on the fecundity or timing of hermaphrodite self-reproduction because if the male fails to mate its fitness is zero, irrespective of what the hermaphrodite does.

Similarly, a hermaphrodite mated to a male with poor sperm will presumably sire a larger fraction of offspring than a hermaphrodite mated to a male with good sperm. Male sperm generally outcompete hermaphrodite sperm
(Lamunyon and Ward 1995), so if it is assumed that the entire difference in $C I_{M}$ is due to reduction in spermcompetitive ability and that wild-type hermaphrodite sperm would be no better competitors than wild-type male sperm (which seems reasonable), then the strength of selection captured in $C I_{M}$ provides an upper bound on the strength of selection acting on the competitive ability of male sperm relative to hermaphrodite sperm. Alas, no such simple approximation is possible with respect to male mating behavior, because the fitness consequences of even the simplest aspect of male behavior, time to mating, depend on the distribution of timing of hermaphrodite selffertilization.

A final consideration is the possibility that the results may be peculiar to the $\mathrm{N} 2$ genotype. $\mathrm{N} 2$ is well-known to be atypical relative to $C$. elegans wild isolates in many respects, conspicuously including the poor mating ability of N2 males (Sterken et al. 2015). Since N2 was maintained by self-fertilization for thousands of generations, male function has already suffered the results of a protracted period of mutation accumulation. If mutations have consistently epistatic effects, conclusions drawn from N2 may be misleading with respect to selection in the wild.

\section{Data archiving}

Data available from the Dryad Digital Repository: http://dx. doi.org/10.5061/dryad.2ng4k

Acknowledgements We thank D. Blanton, W. Bour, M. Damas, T. Keller, L. Levy, D. Ostrow and N. Phillips for assistance in the lab. $C$. elegans strains were graciously provided by K. Choe (UF) and the Caenorhabditis Genetics Center. Two anonymous reviewers provided helpful comments. We especially thank H. Teotónio for his very helpful and detailed comments, and for providing the data reported in Teotónio et al. (2006). Support was provided by NIH grants R01GM107227 to CFB and E. Andersen, and S1010OD012006 to CFB.

\section{Compliance with ethical standards}

Conflict of Interest The authors declare no conflict of interest.

\section{References}

Andersen EC, Gerke JP, Shapiro JA, Crissman JR, Ghosh R, Bloom JS et al. (2012) Chromosome-scale selective sweeps shape Caenorhabditis elegans genomic diversity. Nat Genet 44(3): 285-U283

Anderson JL, Morran LT, Phillips PC (2010) Outcrossing and the maintenance of males within C. elegans populations. Journal of Heredity 101:S62-S74

Baer CF, Phillips N, Ostrow D, Avalos A, Blanton D, Boggs A et al. (2006) Cumulative effects of spontaneous mutations for fitness in Caenorhabditis: Role of genotype, environment and stress. Genetics 174(3):1387-1395

Baer CF, Shaw F, Steding C, Baumgartner M, Hawkins A, Houppert A et al. (2005) Comparative evolutionary genetics of spontaneous 
mutations affecting fitness in rhabditid nematodes. Proceedings of the National Academy of Sciences of the United States of America 102(16):5785-5790

Barton NH (1990) Pleiotropic models of quantitative variation. Genetics 124(3):773-782

Barton NH, Briggs DEG, Eisen JA, Goldstein DB, Patel NH (2007) Evolution. Cold Spring Harbor Laboratories Press. Cold Spring Harbor, NY, USA

Bateman AJ (1948) Intra-sexual selection in Drosophila. Heredity 2(3):349-368

Charlesworth B (2015) Causes of natural variation in fitness: Evidence from studies of Drosophila populations. Proceedings of the National Academy of Sciences of the United States of America 112(6):1662-1669

Charnov EL (1982) The Theory of Sex Allocation. Princeton University Press, Princeton, NJ, USA

Clark DV, Rogalski TM, Donati LM, Baillie DL (1988) The Unc-22 (IV) region of Caenorhabditis elegans: Genetic analysis of lethal mutations. Genetics 119(2):345-353

Cutter AD (2008) Divergence times in Caenorhabditis and Drosophila inferred from direct estimates of the neutral mutation rate. Mol Biol Evol 25(4):778-786

Cutter AD, Wasmuth JD, Washington NL (2008) Patterns of molecular evolution in Caenorhabditis preclude ancient origins of selfing. Genetics 178(4):2093-2104

Davies SK, Leroi AM, Burt A, Bundy J, Baer CF (2016). The mutational structure of metabolism in Caenorhabditis elegans. Evolution 70:2239-2246.

Denver DR, Dolan PC, Wilhelm LJ, Sung W, Lucas-Lledo JI, Howe DK et al. (2009) A genome-wide view of Caenorhabditis elegans base-substitution mutation processes. Proceedings of the National Academy of Sciences of the United States of America 106(38):16310-16314

Denver DR, Morris K, Lynch M, Thomas WK (2004) High mutation rate and predominance of insertions in the Caenorhabditis elegans nuclear genome. Nature 430(7000):679-682

Denver DR, Wilhelm LJ, Howe DK, Gafner K, Dolan PC, Baer CF (2012) Variation in base-substitution mutation in experimental and natural lineages of Caenorhabditis nematodes. Genome Biology and Evolution 4(4):513-522

Dong CF, Dolke F, von Reuss SH (2016) Selective MS screening reveals a sex pheromone in Caenorhabditis briggsae and speciesspecificity in indole ascaroside signalling. Org Biomol Chem 14(30):7217-7225

Drake JW, Charlesworth B, Charlesworth D, Crow JF (1998) Rates of spontaneous mutation. Genetics 148(4):1667-1686

Efron B, Tibshirani RJ (1993) An Introduction to the Bootstrap. Chapman and Hall, New York

Eigen M (1971) Self-organization of matter and evolution of biological macromolecules. Naturwissenschaften 58(10):465 -\&

Falconer DS (1989) Quantitative Genetics, 3rd edn. Longman Scientific and Technical, Essex, UK

Fisher RA (1930) The Genetical Theory of Natural Selection. Clarendon Press, Oxford

Fry JD (2004) Estimation of genetic variances and covariances by restricted maximum likelihood using PROC MIXED. In: Saxton AM (ed.) Genetic Analysis of Complex Traits Using SAS. SAS Institute, Inc., Cary, NC, pp 11-34

Fry JD, Heinsohn SL (2002) Environment dependence of mutational parameters for viability in Drosophila melanogaster. Genetics 161(3):1155-1167

He F (2011) Making males of C. elegans. Bio-protocol Bio 101:e58

Herman RK (2005) Wormbook. Community TCeR (ed.).

Hodgkin J, Doniach T (1997) Natural variation and copulatory plug formation in Caenorhabditis elegans. Genetics 146(1):149-164
Houle D, Hughes KA, Hoffmaster DK, Ihara J, Assimacopoulos S, Canada D et al. (1994) The effects of spontaneous mutation on quantitative traits. 1. Variances and covariances of life-history traits. Genetics 138(3):773-785

Houle D, Morikawa B, Lynch M (1996) Comparing mutational variabilities. Genetics 143(3):1467-1483

Igic B, Busch JW (2013) Is self-fertilization an evolutionary dead end? New Phytol 198(2):386-397

Kenward MG, Roger JH (1997) Small sample inference for fixed effects from restricted maximum likelihood. Biometrics 53(3): 983-997

Kiontke KC, Felix MA, Ailion M, Rockman MV, Braendle C, Penigault JB et al. (2011) A phylogeny and molecular barcodes for Caenorhabditis, with numerous new species from rotting fruits. BMC Evol Biol 11:339

Lamunyon CW, Ward S (1995) Sperm precedence in a hermaphroditic nematode (Caenorhabditis elegans) is due to competitive ability of male sperm. Experientia 51(8):817-823

Loewe L, Cutter AD (2008). On the potential for extinction by Muller's Ratchet in Caenorhabditis elegans. BMC Evol Biol $8: 125$

Luria SE, Delbrück M (1943) Mutations of bacteria from virus sensitivity to virus resistance. Genetics 28(6):491-511

Lynch M, Walsh B (1998) Genetics and Analysis of Quantitative Traits. Sinauer, Sunderland, MA

Mallet MA, Bouchard JM, Kimber CM, Chippindale AK (2011) Experimental mutation-accumulation on the $\mathrm{X}$ chromosome of Drosophila melanogaster reveals stronger selection on males than females. BMC Evol Biol 11:156

Mallet MA, Kimber CM, Chippindale AK (2012) Susceptibility of the male fitness phenotype to spontaneous mutation. Biol Lett $8(3)$ : 426-429

Morton NE, Crow JF, Muller HJ (1956) An estimate of the mutational damage in man from data on consanguinous marriages. Proceedings of the National Academy of Sciences of the United States of America 42(11):855-863

Mukai T, Chigusa SI, Crow JF, Mettler LE (1972) Mutation rate and dominance of genes affecting viability in Drosophila melanogaster. Genetics 72(2):335-355

Murray RL, Kozlowska JL, Cutter AD (2011) Heritable determinants of male fertilization success in the nematode Caenorhabditis elegans. BMC Evol Biol 11:99

Ohta T (1973) Slightly deleterious mutant substitutions in evolution. Nature 246(5428):96-98

Rice WR, Chippindale AK (2001) Intersexual ontogenetic conflict. J Evol Biol 14(5):685-693

Rosenbluth RE, Cuddeford C, Baillie DL (1983) Mutagenesis in Caenorhabditis elegans. 1. A rapid eukaryotic mutagen test system using the reciprocal translocation et1(III-V). Mutat Res 110(1):39-48

Rowe L, Houle D (1996) The lek paradox and the capture of genetic variance by condition dependent traits. Proceedings of the Royal Society B-Biological Sciences 263 (1375):1415-1421

Schedl T, Kimble J (1988) Fog-2, a germ-line-specific sex determination gene required for hermaphrodite spermatogenesis in Caenorhabditis elegans. Genetics 119(1):43-61

Schultz ST, Lynch M (1997) Mutation and extinction: The role of variable mutational effects, synergistic epistasis, beneficial mutations, and degree of outcrossing. Evolution 51(5): 1363-1371

Shabalina SA, Yampolsky LY, Kondrashov AS (1997) Rapid decline of fitness in panmictic populations of Drosophila melanogaster maintained under relaxed natural selection. Proceedings of the National Academy of Sciences of the United States of America 94(24):13034-13039 
Sharp NP, Agrawal AF (2013) Male-biased fitness effects of spontaneous mutations in Drosophila melanogaster. Evolution 67(4): 1189-1195

Shaw RG, Byers DL, Darmo E (2000) Spontaneous mutational effects on reproductive traits of Arabidopsis thaliana. Genetics 155(1): 369-378

Sterken MG, Snoek LB, Kammenga JE, Andersen EC (2015) The laboratory domestication of Caenorhabditis elegans. Trends in Genetics 31(5):224-231

Stewart AD, Phillips PC (2002) Selection and maintenance of androdioecy in Caenorhabditis elegans. Genetics 160(3):975-982

Teotonio H, Carvalho S, Manoel D, Roque M, Chelo IM (2012) Evolution of Outcrossing in Experimental Populations of Caenorhabditis elegans. PLoS One 7(4)
Teotonio H, Manoel D, Phillips PC (2006) Genetic variation for outcrossing among Caenorhabditis elegans isolates. Evolution 60(6): 1300-1305

The GLIMMIX Procedure (2013) SAS/STAT 13.1 User's Guide. SAS Institute, Inc., Cary, NC

Vassilieva LL, Hook AM, Lynch M (2000) The fitness effects of spontaneous mutations in Caenorhabditis elegans. Evolution 54(4):1234-1246

Vassilieva LL, Lynch M (1999) The rate of spontaneous mutation for life-history traits in Caenorhabditis elegans. Genetics 151(1): 119-129

Willis JH (1999) The contribution of male-sterility mutations to inbreeding depression in Mimulus guttatus. Heredity $83: 337-346$ 